\title{
Venoarterial extracorporeal membrane oxygenation for right heart failure complicating left ventricular assist device use
}

\author{
Masashi Kai, MD, Gilbert H. L. Tang, MD, MSc, Ramin Malekan, MD, Steven L. Lansman, MD, PhD, and \\ David Spielvogel, MD, Valhalla, NY
}

From $15 \%$ to $25 \%$ of patients have right heart failure (RHF) develop after left ventricular (LV) assist device (LVAD) placement, ${ }^{1}$ significantly increasing morbidity and mortality. ${ }^{2}$ Right ventricular (RV) assist devices (RVADs) may support RV function, but they have significant limitations. We describe a novel management strategy of using peripheral venoarterial extracorporeal membrane oxygenation (ECMO), ${ }^{3}$ configured for easy explantation, that may improve RV recovery and reduce mortality and morbidity.

\section{CLINICAL SUMMARY}

From March 2012 to March 2013, a total of 5 patients with cardiogenic shock and biventricular failure supported by venoarterial ECMO underwent Heartmate II (HM II) LVAD implantation with ECMO support for RHF (Table 1). Preoperative ECMO duration was 7.8 days (3-17 days). Of the 5 patients, 3 had axillary arterial outflow and the 2 with central cannulation had conversion to an arterial outflow graft connected end to side to the HM II outflow graft, which was exteriorized through the right side of the chest (Figure 1).

To optimize systemic output, it is important to balance the HM II and ECMO flows. In the operating room, interventricular septal position was assessed by transesophageal echocardiography, and flows were adjusted to unload the RV sufficiently while avoiding inadequate HM II preload and yet augmenting systemic output. We found that ECMO flows of 2 to $3 \mathrm{~L} / \mathrm{min}$, with the HM II speed set at $8400 \mathrm{rpm}$, will generally achieve this balance. In the intensive care unit, inotrope and vasopressor use can be minimized, which promotes normalization of peripheral vascular resistance and aids RV, pulmonary, and visceral recoveries. We found that adjustments of HM II speed and ECMO flow are rarely necessary; when indicated, output

\footnotetext{
From the Department of Surgery, Section of Cardiothoracic Surgery, New York Medical College, Westchester Medical Center, Valhalla, NY.

Disclosures: Authors have nothing to disclose with regard to commercial support.

Received for publication July 30, 2013; revisions received Sept 19, 2013; accepted for publication Oct 6, 2013; available ahead of print Dec 2, 2013.

Address for reprints: David Spielvogel, MD, Section of Cardiothoracic Surgery, Westchester Medical Center, 100 Woods Rd, Macy 114W, Valhalla, NY (E-mail: SpielvogelD@wcmc.com).

J Thorac Cardiovasc Surg 2014;147:e31-3

$0022-5223 / \$ 36.00$

Copyright (C) 2014 by The American Association for Thoracic Surgery

http://dx.doi.org/10.1016/j.jtcvs.2013.10.040
}

was best augmented by increasing the volume rather than by adjusting the ECMO flow. Heparin was administered to achieve a target activated clotting time of 200 to 240 seconds.

All patients were successfully weaned from ECMO after an average of 4.8 days of support, with no deaths, strokes, or vascular complications. There was 1 in-hospital death. Follow-up was $9.9 \pm 3.3$ months and $100 \%$ complete. Among the 4 patients who survived the HM II implantation hospitalization, 1 underwent cardiac transplant, 1 is currently awaiting transplant, 1 had LV recovery while awaiting HM II explantation, and 1 died of stroke 6 months after HM II placement.

\section{DISCUSSION}

Although RVADs have played an important role for RHF after LVAD implantation, associated mortality remains high, ${ }^{2}$ and a number of factors, such as bleeding, infection, and thromboembolism, may limit their usefulness in this setting.

First, a major disadvantage of an RVAD in this setting is that it does not unload the RV but rather increases RV afterload. In addition, if RVAD flow sufficiently increases pulmonary arterial pressure, it may precipitate or increase pulmonic insufficiency, exacerbating RV distention. On the other hand, ECMO, by bypassing the right side of the heart, does unload the RV and may promote RV healing more efficiently than does an RVAD. A second potential disadvantage of an RVAD is that it can exacerbate pulmonary edema, because it can directly perfuse the lungs at high flows and high pressures. Venoarterial ECMO drops the pulmonary artery pressure by bypassing the lungs and may thereby promote resolution of pulmonary edema. Finally, by augmenting systemic perfusion and improving venous drainage, ECMO may lower the central venous pressure more effectively, optimize end-organ perfusion, and more effectively reverse hepatorenal and cardiorenal syndromes. All our patients with HM IIs who received ECMO support had their hepatic and renal injuries reversed.

A potential disadvantage of ECMO is that it can increase LV afterload. In our cases, however, the patients also had an LVAD, which was better able to handle afterload than a failing LV would be. Another potential limitation of the configuration that we describe here is that 2 pumps perfuse the systemic circulation, increasing the risk of systemic 
TABLE 1. Patient data $(\mathbf{N}=5)$

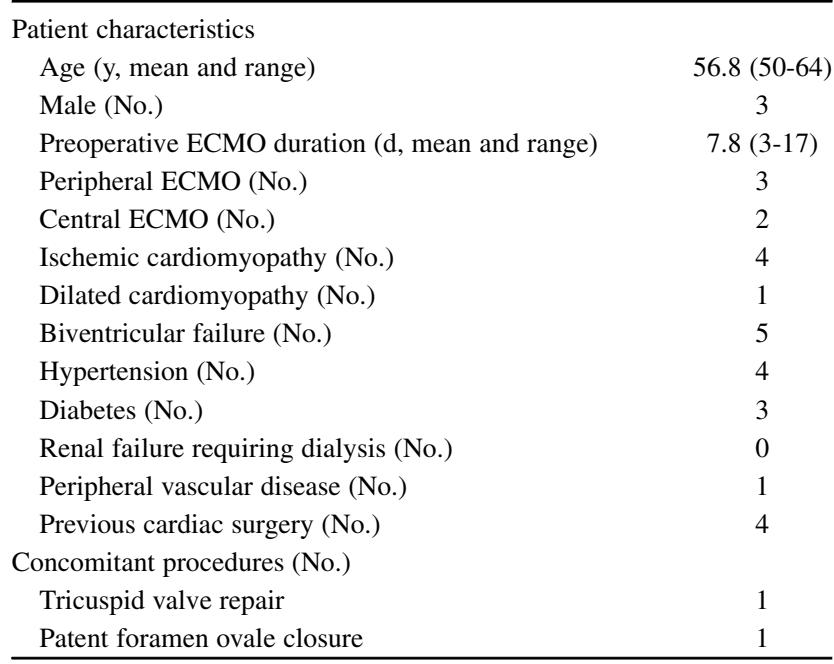

embolization. In this limited series, however, no patients had any embolic events during ECMO support. We did not find patients with HM II placement who were receiving venoarterial ECMO support to have any more bleeding complications and blood product transfusions than did patients with HM II placement alone.

De Silva and coworkers ${ }^{4}$ described a peripheral RVAD technique through an ECMO system to allow closed-chest explantation. Although this approach does facilitate RVAD explantation, it does not confer the advantage of unloading the RV as does ECMO with outflow to the systemic circulation, as described here.

Peripheral venoarterial ECMO for RHF after LVAD implantation was described by Scherer and colleagues. ${ }^{5}$ All 10 of their patients were weaned from ECMO, although 4 patients died while receiving LVAD support. We have also found this approach useful in our patients; however, in cases of (1) central aortic cannulation, (2) subclavian artery stenosis, or (3) axillary artery diameter inadequate for ECMO flow, the strategy of coupling ECMO and LVAD outflow grafts (Figure 1) offers a simple solution for central systemic perfusion.

We believe that this management strategy of venoarterial ECMO configured for easy explantation may improve RV recovery and reduce the postoperative complications and

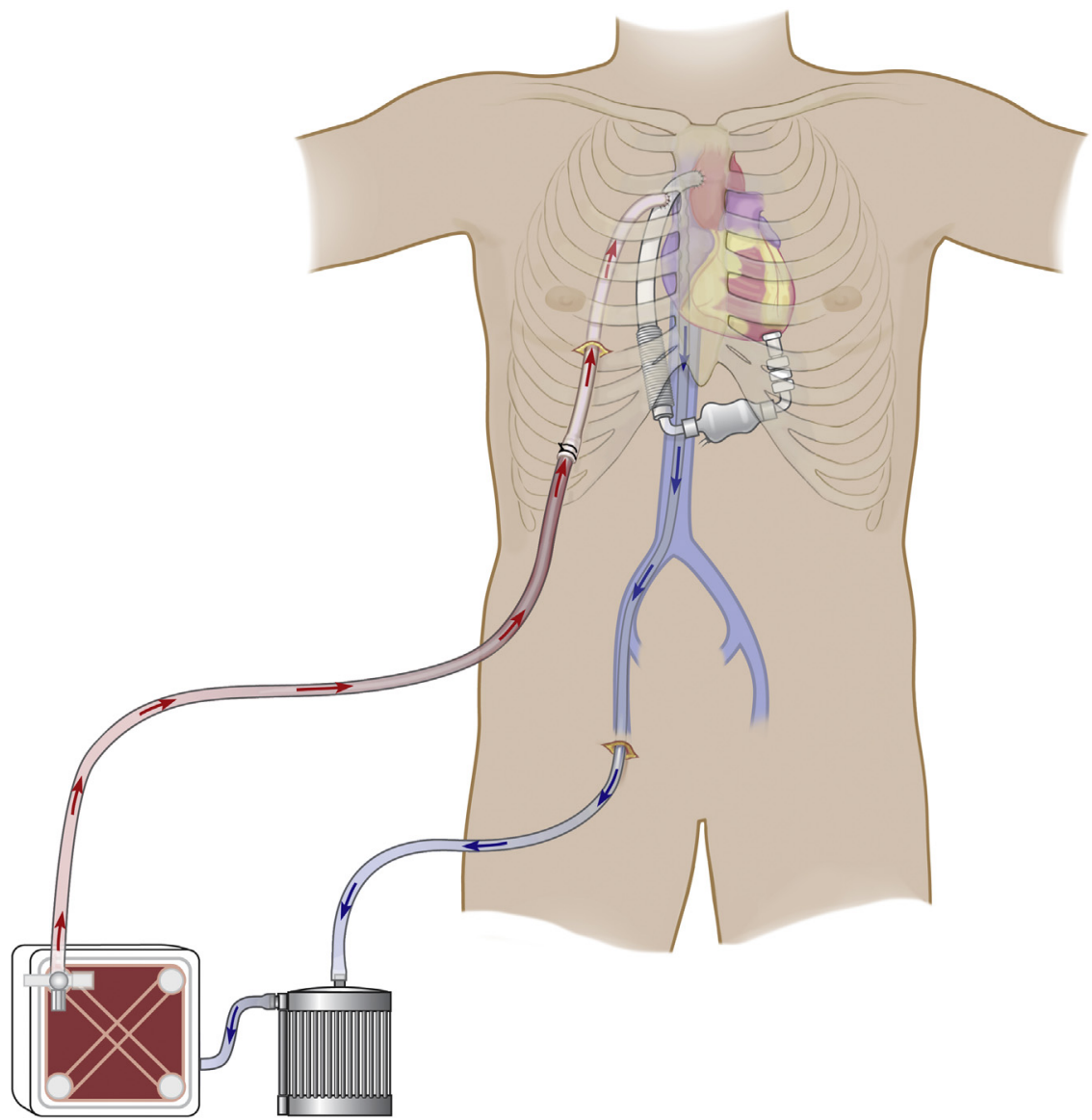

FIGURE 1. Left ventricular assist device with venoarterial extracorporeal membrane oxygenation. The extracorporeal membrane oxygenation outflow is directed to the systemic circulation through an 8-mm Dacron polyester fabric graft, which is tunneled through the right pleural cavity and anastomosed to the left ventricular assist device outflow graft. 
mortality associated with RHF complicating LVAD implantation.

We thank Drs Alan Gass and Gregg Lanier for their contributions to this study.

\section{References}

1. Rich JD. Right ventricular failure in patients with left ventricular assist devices. Cardiol Clin. 2012;30:291-302.

2. Kavarana MN, Pessin-Minsley MS, Urtecho J, Catanese KA, Flannery M, $\mathrm{Oz} \mathrm{MC}$, et al. Right ventricular dysfunction and organ failure in left ventricular assist device recipients: a continuing problem. Ann Thorac Surg. 2002;73 745-50.

3. Tang GH, Malekan R, Kai M, Lansman SL, Spielvogel D. Peripheral venoarterial extracorporeal membrane oxygenation improves survival in myocardial infarction with cardiogenic shock. J Thorac Cardiovasc Surg. 2013;145:e32-3.

4. De Silva RJ, Soto C, Spratt P. Extra corporeal membrane oxygenation as right heart support following left ventricular assist device placement: a new cannulation technique. Heart Lung Circ. 2012;21:218-20

5. Scherer M, Sirat AS, Moritz A, Martens S. Extracorporeal membrane oxygenation as perioperative right ventricular support in patients with biventricular failure undergoing left ventricular assist device implantation. Eur J Cardiothorac Surg. 2011;39:939-44; discussion 944 . 